\title{
Physical activity attenuates postprandial hyperglycaemia in homozygous TBC1D4 loss-of-function mutation carriers
}

\author{
Theresia M. Schnurr ${ }^{1}$ (D) Emil Jørsboe ${ }^{1,2}$ - Alexandra Chadt ${ }^{3,4} \cdot$ Inger K. Dahl-Petersen $^{5,6}$ • Jonas M. Kristensen ${ }^{7}$. \\ Jørgen F. P. Wojtaszewski ${ }^{7}$. Christian Springer ${ }^{3,4}$ • Peter Bjerregaard ${ }^{5}$. Søren Brage ${ }^{8}$. Oluf Pedersen ${ }^{1} \cdot$ Ida Moltke $^{2}$. \\ Niels Grarup ${ }^{1} \cdot$ Hadi Al-Hasani ${ }^{3,4} \cdot$ Anders Albrechtsen $^{2} \cdot$ Marit E. Jørgensen $^{5,6,9} \cdot$ Torben Hansen $^{1}$
}

Received: 30 November 2020 / Accepted: 24 February 2021 / Published online: 29 April 2021

(C) The Author(s) 2021

\begin{abstract}
Aims/hypothesis The common muscle-specific TBC1D4 p.Arg684Ter loss-of-function variant defines a subtype of non-autoimmune diabetes in Arctic populations. Homozygous carriers are characterised by elevated postprandial glucose and insulin levels. Because $3.8 \%$ of the Greenlandic population are homozygous carriers, it is important to explore possibilities for precision medicine. We aimed to investigate whether physical activity attenuates the effect of this variant on $2 \mathrm{~h}$ plasma glucose levels after an oral glucose load.

Methods In a Greenlandic population cohort $(n=2655), 2 \mathrm{~h}$ plasma glucose levels were obtained after an OGTT, physical activity was estimated as physical activity energy expenditure and TBC1D4 genotype was determined. We performed TBC1D4physical activity interaction analysis, applying a linear mixed model to correct for genetic admixture and relatedness.

Results Physical activity was inversely associated with $2 \mathrm{~h}$ plasma glucose levels ( $\beta$ [main effect of physical activity] -0.0033 $[\mathrm{mmol} / 1] /\left[\mathrm{kJ} \mathrm{kg}^{-1} \mathrm{day}^{-1}\right], p=6.5 \times 10^{-5}$ ), and significantly more so among homozygous carriers of the TBC1D4 risk variant compared with heterozygous carriers and non-carriers ( $\beta$ [interaction $]-0.015[\mathrm{mmol} / 1] /\left[\mathrm{kJ} \mathrm{kg}^{-1} \mathrm{day}^{-1}\right], p=0.0085$ ). The estimated effect size suggests that $1 \mathrm{~h}$ of vigorous physical activity per day (compared with resting) reduces $2 \mathrm{~h}$ plasma glucose levels by an additional $\sim 0.7 \mathrm{mmol} / \mathrm{l}$ in homozygous carriers of the risk variant.

Conclusions/interpretation Physical activity improves glucose homeostasis particularly in homozygous TBCID4 risk variant carriers via a skeletal muscle TBC1 domain family member 4-independent pathway. This provides a rationale to implement physical activity as lifestyle precision medicine in Arctic populations.

Data repository The Greenlandic Cardio-Metabochip data for the Inuit Health in Transition study has been deposited at the European Genome-phenome Archive (https://www.ebi.ac.uk/ega/dacs/EGAC00001000736) under accession EGAD00010001428.
\end{abstract}

Keywords Arctic $\cdot$ Gene-environment interaction $\cdot$ Lifestyle therapy $\cdot$ Physical activity $\cdot$ Postprandial hyperglycaemia $\cdot T B C 1 D 4$ loss-of-function

Theresia M. Schnurr and Emil Jørsboe are equal contributors to this work.

Marit E. Jørgensen and Torben Hansen share senior authorship.

Torben Hansen

torben.hansen@sund.ku.dk

1 Novo Nordisk Foundation Center for Basic Metabolic Research, Faculty of Health and Medical Sciences, University of Copenhagen, Copenhagen, Denmark

2 The Bioinformatics Centre, Department of Biology, University of Copenhagen, Copenhagen, Denmark

3 Institute for Clinical Biochemistry and Pathobiochemistry, German Diabetes Center (DDZ), Leibniz Center for Diabetes research at the Heinrich-Heine-University Duesseldorf, Medical Faculty, Duesseldorf, Germany

4 German Center for Diabetes Research (DZD), Duesseldorf, Germany
5 National Institute of Public Health, University of Southern Denmark, Odense, Denmark

6 Steno Diabetes Center Copenhagen, Gentofte, Denmark

7 Section of Molecular Physiology, Department of Nutrition, Exercise and Sports, Faculty of Science, University of Copenhagen, Copenhagen, Denmark

8 Medical Research Council Epidemiology Unit, University of Cambridge, Cambridge, UK

9 Greenland Center for Health Research, University of Greenland, Nuuk, Greenland 


\section{Research in context}

\section{What is already known about this subject?}

- Recently, we identified the common TBC1D4 p.Arg684Ter loss-of-function variant in the Greenlandic population

- Homozygous carriers of the TBC1D4 risk allele are characterised by postprandial hyperglycaemia, markedly increased odds of type 2 diabetes (OR 10.3), decreased expression of GLUT4 in skeletal muscle and decreased insulin-stimulated glucose uptake

- The effect of lifestyle behaviours (i.e. physical activity) on metabolic health may vary between individuals depending on genetic variation

\section{What is the key question?}

- Is the effect of the common TBC1D4 p.Arg684Ter loss-of-function variant on $2 \mathrm{~h}$ plasma glucose levels attenuated by physical activity?

What are the new findings?

- We found that self-reported physical activity associates with lower $2 \mathrm{~h}$ plasma glucose levels, particularly in homozygous TBC1D4 risk variant carriers

- We estimated that $1 \mathrm{~h}$ of vigorous physical activity per day reduces $2 \mathrm{~h}$ plasma glucose levels by an additional $\sim 0.4-0.7 \mathrm{mmol} / \mathrm{l}$ in homozygous TBC1D4 risk variant carriers compared with non-carriers and heterozygous carriers

\section{How might this impact on clinical practice in the foreseeable future?}

- This finding provides a rationale to implement physical activity as a first step towards lifestyle precision medicine in homozygous TBC1D4 mutation carriers, who make up 3.8\% of the Arctic Inuit population

\section{Abbreviations \\ MET Metabolic equivalent task \\ RabGAP Rab-GTPase-activating protein \\ TBC1D4 TBC1 domain family member 4}

\section{Introduction}

Type 2 diabetes is a growing global health problem and its prevalence is also rapidly increasing in Greenland and in other Arctic populations [1-3]. Recently, a common TBC1D4 p.Arg684Ter loss-of-function variant that defines a specific subtype of non-autoimmune diabetes characterised by elevated postprandial glucose levels has been described. The variant was identified initially in the Greenlandic population (minor allele frequency of $17 \%$, and $3.8 \%$ are homozygous carriers) and later described in the North American Inuit population with similar allele frequency [4, 5]. Homozygous carriers are characterised by postprandial hyperglycaemia (on average these individuals had $3.8 \mathrm{mmol} / \mathrm{l}$ higher $2 \mathrm{~h}$ plasma glucose during an OGTT), impaired glucose tolerance and 10.3-fold increased odds of developing type 2 diabetes [4]. The mutation is estimated to account for more than $10 \%$ of all cases of type 2 diabetes in Greenland and other Artic Inuit populations $[4,5]$. Based on the variant's high frequency and the clinical characterisation of homozygous TBC1D4 risk variant carriers, it has been suggested that diagnostic strategies for type 2 diabetes should include $2 \mathrm{~h}$ OGTT and/or TBC1D4 genotype risk stratification in Arctic populations [5-7].

There is a plausible pathophysiological explanation for the observed clinical characteristics of homozygous TBC1D4 risk variant carriers. TBC1D4 encodes a Rab-GTPase-activating protein (RabGAP) that represents a key factor in the insulinstimulated GLUT4 translocation process [8]. Previously, we observed decreased GLUT4 content in skeletal muscle of homozygous carriers and speculate that this likely contributes to postprandial hyperglycaemia via a possible lowered glucose uptake in skeletal muscle [4]. Experiments in mice have shown that knocking down Tbcld4 causes a decrease in basal plasma glucose and a decrease in insulin-stimulated glucose uptake in muscle and adipose tissue [9]. Regular exercise training has been shown to regulate the phosphorylation of TBC1 domain family member 4 (TBC1D4) in skeletal muscle from individuals with type 2 diabetes, indicating a role for RabGAP in the insulin-sensitising effects of exercise training [10]. Importantly, regular physical activity is widely accepted as a useful means for both the prevention and treatment of metabolic diseases $[11,12]$ but the underlying mechanisms of the beneficial impact of physical activity on metabolic health are not yet fully understood.

It is not currently known whether daily physical activity especially benefits indigenous Arctic individuals with a muscular TBC1D4 deficiency. Such knowledge might provide a powerful treatment option to effectively lower 
postprandial glucose levels and prevent type 2 diabetes in these populations and might provide insights into molecular mechanisms. Motivated by this, our main aim was to estimate the effect of daily physical activity on the association between the common TBC1D4 p.Arg684Ter loss-of-function variant and postprandial plasma glucose levels during a 2 h OGTT in Greenlandic individuals. As secondary outcomes, we also examined whether daily physical activity reduces the odds of developing type 2 diabetes and has beneficial effects on other cardiometabolic traits in homozygous carriers of this loss-offunction variant. To our knowledge, this is the first study investigating the effect of gene-lifestyle interactions on cardiometabolic traits in Artic populations.

\section{Methods}

Study population The Greenlandic cohort $(n=3115)$, also known as the Inuit Health in Transition study, is a population-based study of around 9\% of the adult Greenlandic population. Data was collected after inviting a random sample of adults aged $\geq 18$ years to participate in the population health survey in Greenland in 2005-2010. Pregnant women were not enrolled. Details about the health survey and data collection method are described in detail elsewhere [2]. Prior to participation, informed consent was obtained from all individuals included into the study. The study was approved by the Commission for Scientific Research in Greenland (project 2011-13, ref. no. 2011-056978, and project 2013-13, ref.no. 2013-090702) and was conducted in accordance with the principles of the Declaration of Helsinki.

\section{Cardiometabolic traits and definition of type 2 diabetes All} participants without known diabetes (at time of the examination) underwent a standardised $75 \mathrm{~g}, 2 \mathrm{~h}$ OGTT after an overnight fast of at least $8 \mathrm{~h}$. Plasma glucose was analysed with the Hitachi 912 system (Roche Diagnostics). Measurements of secondary tested cardiometabolic traits are described in the electronic supplementary material (ESM) Methods. Type 2 diabetes was classified according to WHO criteria [13].

Physical activity assessment Information on daily physical activity was collected using an interviewer-administrated version of the International Physical Activity Questionnaire (long version) that was modified and adapted to Arctic living conditions ( $n=3004$ individuals of the entire cohort) as described in ESM Methods. Physical activity energy expenditure was calculated [14]. This questionnaire was found to be a valid measure for overall physical activity energy expenditure among adult Inuit in Greenland [14]. We based our primary main analysis on questionnaire-derived physical activity for enhanced power of our statistical analysis since this allowed us to include as many individuals as possible with valid information on physical activity, TBC1D4 p.Arg684Ter genotype and $2 \mathrm{~h}$ plasma glucose concentrations $(n=2655)$. We performed sensitivity analysis in a subset of individuals $(n=$ 1388) where physical activity energy expenditure was also assessed objectively by combined heart rate and acceleration monitors (ActiHeart; CamNTech, Cambridge, UK) to evaluate whether magnitude and direction of interaction effects were comparable between subjectively and objectively assessed physical activity. We included individuals with a monitor wear time of at least $48 \mathrm{~h}(n=1544$ individuals in the entire cohort) for this sensitivity analysis. Due to study logistics, limited time at each study location and a finite stock of monitors, not all participants were given an ActiHeart monitor and the length of recordings from some participants was shorter $[14,15]$. A full description of the populationspecific calibration of the heart rate-energy equation and assessment of physical activity energy expenditure is documented elsewhere [14-16]. We translated the effect sizes derived from the interaction models ( $\beta$ [interaction]) into aspects of intensity and type of activity to ensure relevance in the Greenlandic cohort. For this, we derived metabolic equivalent task (MET) activities based on the definition by Ainsworth et al [17] and the assumption that an increase in physical activity energy expenditure of $10 \mathrm{~kJ} \mathrm{~kg}^{-1}$ day ${ }^{-1}$ is equivalent to about $1 \mathrm{~h}$ intensity of 3.5 MET per day (e.g. moderate-paced walking at 2.8-3.2 miles per hour when walking for transportation on a firm surface) compared with spending this hour at rest. We also derived the equivalent to a highintensity activity of 9.5 MET per day commonly performed by Greenlanders, namely hiking with hunting gear, compared with spending this hour at rest [17].

Genotyping Participants from the Greenlandic cohort $(n=$ 3115) were genotyped by the Cardio-Metabochip, a custom iSelect genotyping array of 196,725 SNPs for genetic studies of metabolic, cardiovascular and anthropometric traits [18], on the Illumina HiScan platform (Illumina, San Diego, CA, USA). Genotypes were called using the GenCall module (version 1.9.4) of GenomeStudio software (version 2011.1; Illumina). During quality control using PLINK software tools (version 1.9; https://www.cog-genomics.org/plink/) [19] we removed a few individuals with mislabelled sex and high rate of missing data ( $>2 \%$ of all SNPs with a minor allele frequency $>0.01$ ), leaving 3074 individuals for which array genotyping was successful accomplished. The p.Arg684Ter nonsense polymorphism in $T B C 1 D 4$, rs61736969, was genotyped in all participants $(n=3040)$ by applying the KASP genotyping assay (LGC Genomics, UK).

Statistical analysis Overall, 2655 individuals had complete information on questionnaire-derived physical activity, TBC1D4 p.Arg684Ter genotype and $2 \mathrm{~h}$ plasma glucose levels, and hence were included in our primary analysis 
(ESM Fig. 1). We tested for a gene-environment interaction effect between TBC1D4 p.Arg684Ter genotype and physical activity on $2 \mathrm{~h}$ plasma glucose using a linear mixed model implemented in GMMAT (version 1.0.3) [20]. We used a linear mixed model to correct for admixture and relatedness (see Eq. 1 in ESM Methods). We applied a gene-environment model with $2 \mathrm{~h}$ plasma glucose as response, with main effect terms for physical activity and TBC1D4 p.Arg684Ter genotype, and an additional effect term for interaction between physical activity and TBC1D4 p.Arg684Ter. Our group has shown previously that the effect of the TBC1D4 p.Arg684Ter risk variant on $2 \mathrm{~h}$ plasma glucose levels is almost entirely recessive but there is some effect of being heterozygous [4]. In the primary analytical model, we therefore modelled the TBC1D4 p.Arg684Ter effects as recessive. Including the interaction effect term in the model allowed us to test if the effect of physical activity is different for homozygous TBC1D4 p.Arg684Ter risk variant carriers compared with heterozygous carriers and non-carriers and allowed us to estimate the size of this difference ( $\beta$ [interaction]).

Quantitative traits were transformed using sex-specific rankbased inverse normal transformations to ensure that they follow a normal distribution as assumed in the applied model. SEs, effect sizes (in SD) and $p$ values are reported from transformed analyses. We also report effect sizes from analyses of untransformed traits. We applied the Wald test implemented in GMMAT [20] for our primary analysis (see Eq. 1 in ESM Methods), assuming a significance level of 0.05 . We also explored interaction analyses of TBC1D4 with physical activity on additional cardiometabolic traits, assuming a significance level of 0.05 (applying Eq. 1, ESM Methods). Furthermore, we tested for a TBC1D4-physical activity interaction on type 2 diabetes using a logistic model implemented in GMMAT (ESM Methods) [20]. We excluded individuals with known diabetes at the date of examination from all analyses and individuals taking lipid-lowering medications were removed in analysis of lipid measures.

Finally, to compare values of $2 \mathrm{~h}$ plasma glucose levels for different physical activity levels for non-carriers, heterozygous carriers and homozygous carriers of the TBC1D4 p.Arg684Ter risk variant, we predicted values for each of the three possible genotypes for a range of physical activity levels. For this, we used the primary analytical model with the effect sizes estimated based on untransformed $2 \mathrm{~h}$ plasma glucose levels. To obtain predictions relative to an average non-carrier individual with a physical activity level of $50 \mathrm{~kJ} \mathrm{~kg}^{-1} \mathrm{day}^{-1}$, we subtracted $50 \mathrm{~kJ} \mathrm{~kg}^{-1}$ day $^{-1}$ multiplied by $\beta_{1}(\beta[$ main effect for physical activity]) from all predicted values.

\section{Results}

Clinical characteristics of the Greenlandic individuals included in the presented analyses are shown in ESM Table 1. We found that homozygous TBC1D4 p.Arg684Ter variant carriers had highly elevated levels of $2 \mathrm{~h}$ plasma glucose compared with non-carriers ( $\beta$ [main effect of being homozygous $T B C 1 D 4$ risk variant carrier] $=4.2 \mathrm{mmol} / 1, p=1.2 \times$ $\left.10^{-40}, n=2655\right)$, which is consistent with our previous observation [4]. Furthermore, physical activity was inversely associated with $2 \mathrm{~h}$ plasma glucose levels ( $\beta$ [main effect of physical activity $]=-0.0033[\mathrm{mmol} / \mathrm{l}] /\left[\mathrm{kJ} \mathrm{kg}^{-1} \mathrm{day}^{-1}\right], p=6.5 \times$ $10^{-5}, n=2655$, Fig. 1a).

The effect of physical activity on $2 \mathrm{~h}$ plasma glucose levels was significantly greater among homozygous carriers of the TBC1D4 variant compared with heterozygous carriers and non-carriers $(\beta[$ interaction $]=-0.015[\mathrm{mmol} / 1] /$ $\left[\mathrm{kJ} \mathrm{kg}^{-1} \mathrm{day}^{-1}\right], p=0.0085, n=2655$; Fig. $1 \mathrm{~b}$, Table 1 and ESM Fig. 2a). Additional adjustment for BMI did not change the magnitude of interaction $(\beta[$ interaction $]=-0.015$ $\left.[\mathrm{mmol} / 1] /\left[\mathrm{kJ} \mathrm{kg}^{-1} \mathrm{day}^{-1}\right], p=0.011, n=2635\right)$. Importantly, we confirmed the magnitude and direction of the interaction effect in the subset of individuals with available information on objectively assessed physical activity $\left(\beta[\right.$ interaction $]=-0.024[\mathrm{mmol} / 1] /\left[\mathrm{kJ} \mathrm{kg}^{-1} \mathrm{day}^{-1}\right], p=0.45$, $n=1388$; Table 1 ). We did not find any significant difference in physical activity energy expenditure when comparing homozygous TBC1D4 p.Arg684Ter carriers, heterozygous carriers and non-carriers in the Greenlandic population $(p=$ $0.65)$. Additional adjustment of the primary analytical model for total energy consumption did not change the magnitude of $\beta$ (interaction) in individuals with available information on total energy consumption (data not shown).

To be clinically useful, it is important to translate the effect sizes derived from the interaction models ( $\beta$ [interaction]) into real-life settings. Our results suggest that for every $10 \mathrm{~kJ} \mathrm{~kg}^{-1}$ day $^{-1}$ increase in physical activity energy expenditure, concentrations of $2 \mathrm{~h}$ plasma glucose were reduced by an additional $\sim 0.2 \mathrm{mmol} / 1$ in homozygous TBC $1 D 4$ p.Arg684Ter carriers compared with heterozygous carriers and non-carriers (Table 1). This difference in physical activity corresponds to about $1 \mathrm{~h}$ of activity at an intensity of 3.5 MET per day (e.g. moderate-paced walking) compared with spending this hour at rest [17]). More extremely, $1 \mathrm{~h}$ daily of a highintensity activity commonly performed by Greenlanders (e.g. hiking with hunting gear), compared with spending this hour at rest [17], decreased $2 \mathrm{~h}$ plasma glucose levels by an additional $\sim 0.4-0.7 \mathrm{mmol} / 1$ in homozygous TBC $1 D 4$ p.Arg684Ter carriers compared with heterozygous carriers and non-carriers (based on self-reported and objective assessed physical activity, respectively; Table 1).

We also investigated the effect of the TBC $1 D 4$ p.Arg684Ter-physical activity interaction on $2 \mathrm{~h}$ plasma glucose levels under a standard additive interaction model (ESM Methods) and found similar or stronger interaction effects compared with the applied primary recessive interaction model. Remarkably, the additive model showed a 


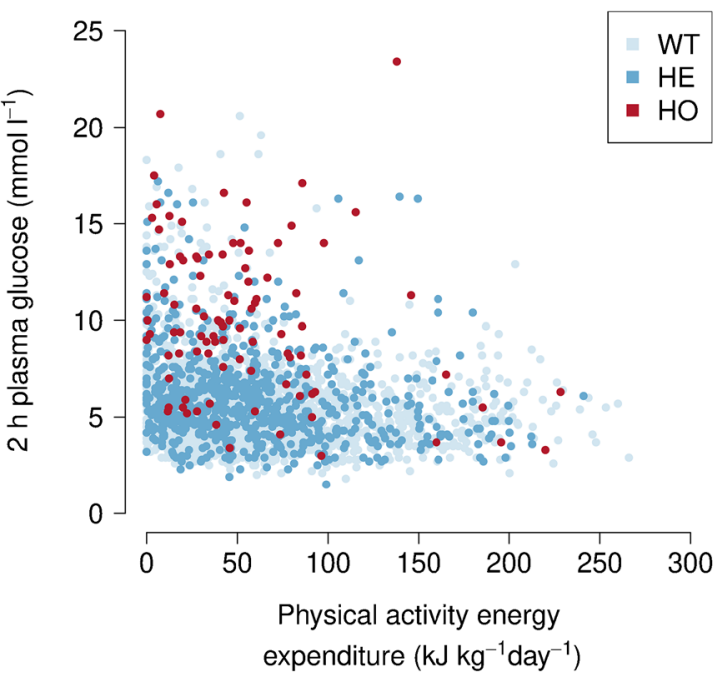

Fig. 1 Physical activity attenuates the effect of the common musclespecific TBC1D4 p.Arg684Ter loss-of-function variant on $2 \mathrm{~h}$ plasma glucose levels in 2655 Greenlanders. (a) Raw data points (physical activity measured as physical activity energy expenditure on the $x$-axis and $2 \mathrm{~h}$ plasma glucose levels on the $y$-axis) stratified by TBC1D4 p.Arg684Ter genotype. (b) Illustration of the TBC1D4-physical activity interaction effect on $2 \mathrm{~h}$ plasma glucose levels relative to a standard individual in Greenlanders. For each individual, the respective mean physical activity energy expenditure per day is plotted on the $x$-axis. The $y$-axis displays

significant interaction effect in the sensitivity analysis of the subset including individuals with objectively measured physical activity ( $p=0.027$, Table 1$)$. To ensure that the results of our primary analysis were not confounded by admixture, we stratified individuals included into the analysis by different degrees of European admixture $(0-5 \%, 5-20 \%, 20-35 \%$ and $>35 \%$ European admixture) and performed interaction analysis by applying the primary model to each subgroup separately. None of the interaction effects for the different admixture groups was significantly in the opposite direction, when taking the uncertainty of the estimates into account, indicating that the presented results are not confounded by population structure (ESM Fig. 2b). Furthermore, we did not observe inflation of our test statistic ( $\lambda=0.82$, ESM Fig. 2c), confirming that the applied linear mixed model satisfactorily accounts for admixture and relatedness among the included individuals.

While our primary aim was to investigate the effect of the TBC1D4-physical activity interaction on $2 \mathrm{~h}$ plasma glucose, our second aim was to investigate the effect of the interaction on the probability of having type 2 diabetes. We included 240 individuals with type 2 diabetes (of these, 38 individuals were homozygous TBC1D4 p.Arg684Ter variant carriers) and 1969 individuals with normal glucose tolerance as a control group (of these, 28 individuals were homozygous TBC1D4 p.Arg684Ter variant carriers) in a logistic model. We found indication of an interaction effect, suggesting an attenuation in the association between the TBC1D4 p.Arg684Ter risk variant and odds of type 2 diabetes per $\mathrm{kJ} \mathrm{kg}^{-1}$ day $^{-1}$ unit increase in

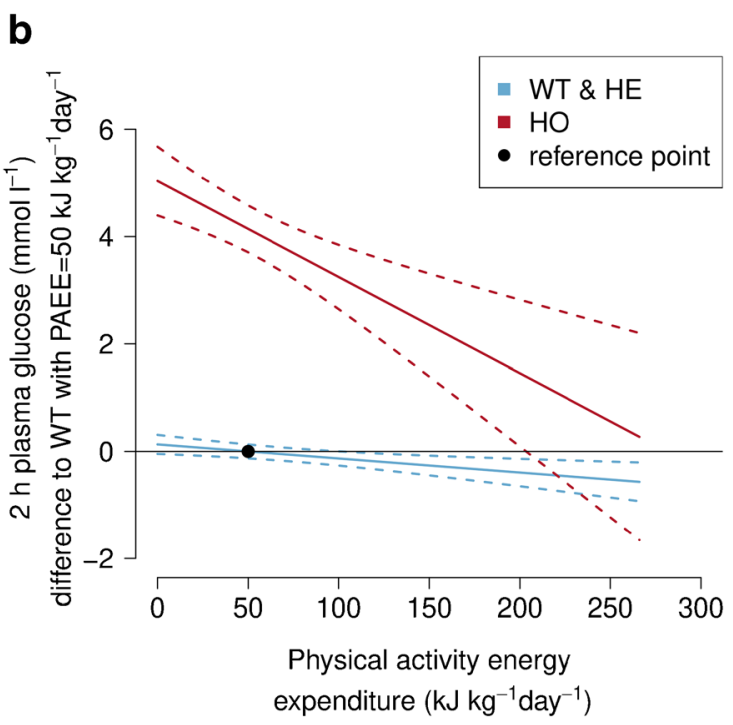

the difference in $2 \mathrm{~h}$ plasma glucose levels for each individual compared with a standard individual (defined as non-carrier having an assumed physical activity energy expenditure of $50 \mathrm{~kJ} \mathrm{~kg}^{-1}$ day ${ }^{-1}[\sim$ median of the cohort, illustrated by the black circle]). The predictions were performed using the estimated effect sizes from the primary analytical model and 95\% CIs are shown. HE, heterozygous TBC1D4 p.Arg684Ter variant carriers; $\mathrm{HO}$, homozygous $T B C 1 D 4$ p.Arg684Ter variant carriers; PAEE, physical activity energy expenditure; WT, non-carriers

daily physical activity (OR 0.99 [95\% CI 0.97, 1.00], $p=$ $0.11)]$. To put this into context, the probability of having type 2 diabetes for a standard individual (40-year-old, male sex) who was a homozygous TBC1D4 risk variant carrier and had an assumed physical activity energy expenditure of $50 \mathrm{~kJ} \mathrm{~kg}^{-1}$ day $^{-1}$ (approximate median of the cohort) was 0.44 . Notably, the probability for type 2 diabetes was reduced to 0.40 with an additional $1 \mathrm{~h}$ of moderate activity $\left(60 \mathrm{~kJ} \mathrm{~kg}^{-1}\right.$ day $\left.^{-1}\right)$ and it was reduced to 0.35 with an additional $1 \mathrm{~h}$ of vigorous activity $\left(77 \mathrm{~kJ} \mathrm{~kg}^{-1}\right.$ day $\left.^{-1}\right)$ per day (ESM Fig. 3a). We did not observe inflation of test statistic in the analysis based on the logistic model $(\lambda=1.11$, ESM Fig. $3 b)$.

Lastly, we investigated the effect of the TBC1D4-physical activity interaction on other cardiometabolic traits and found nominally significant effects on waist/hip ratio $\left(\beta[\right.$ interaction $]=-0.00026 /\left[\mathrm{kJ} \mathrm{kg}^{-1}\right.$ day $\left.^{-1}\right], p=0.047, n=$ 2872; ESM Table 2) and insulin sensitivity index $\left(\beta[\right.$ interaction $]=0.0024 /\left[\mathrm{kJ} \mathrm{kg}^{-1} \mathrm{day}^{-1}\right], p=0.045, n=$ 2634; ESM Table 2).

\section{Discussion}

In the present study of the Greenlandic population, we found that homozygous carriers of the common muscle-specific TBC1D4 p.Arg684Ter loss-of-function variant benefit from physical activity, as shown by lower postprandial plasma 


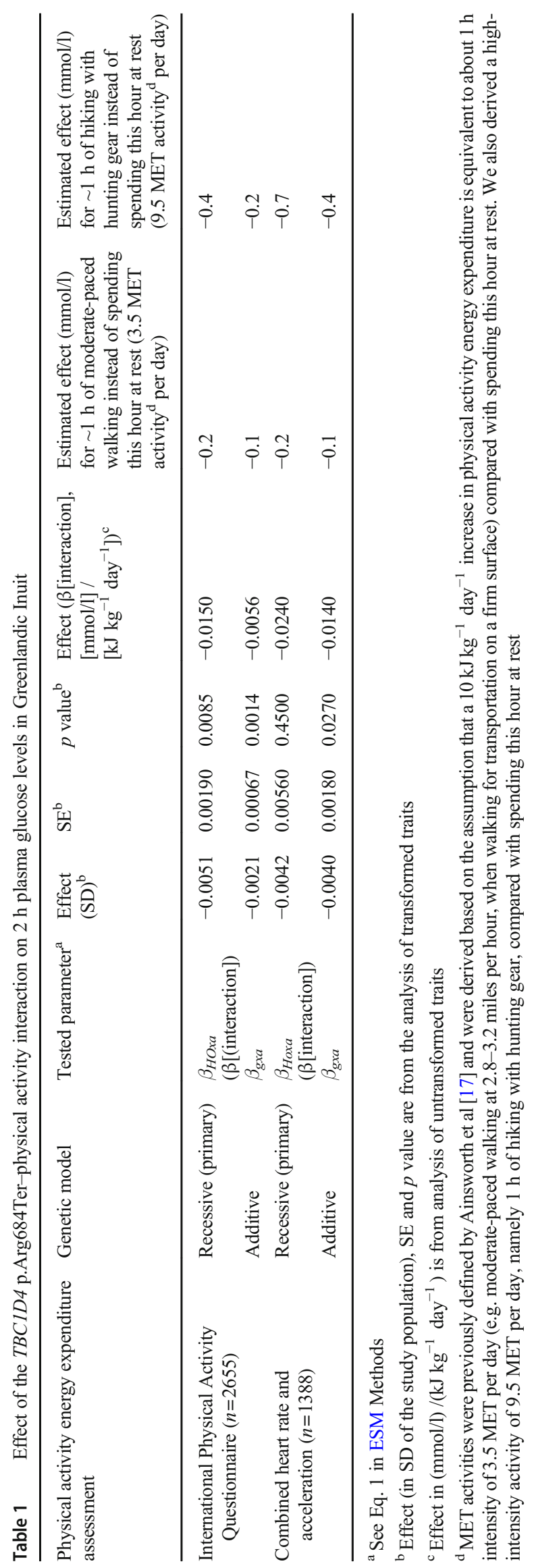

亚 Springer 
glucose levels after an oral glucose load and reduced probability for developing type 2 diabetes. We also found that the effect of physical activity is markedly greater for these carriers.

In fact, the effect size of the gene-environment interaction between the common TBC1D4 p.Arg684Ter loss-of-function variant and physical activity on $2 \mathrm{~h}$ plasma glucose levels in Greenlanders is remarkably large compared with previously identified gene-environment interactions for complex human traits [21-23]. Previous studies in individuals of European ancestry have examined interactions of type 2 diabetesassociated genetic variants and physical activity on metabolic disease traits with mixed results [24-26]. Here, we report that $1 \mathrm{~h}$ of moderate-paced walking or $1 \mathrm{~h}$ of high-intensity activity lowers $2 \mathrm{~h}$ plasma glucose levels by an additional $\sim 0.2 \mathrm{mmol} / 1$ and up to $\sim 0.7 \mathrm{mmol} / \mathrm{l}$, respectively, compared with spending this hour at rest. To place our observed effect sizes of the TBC1D4-physical activity interaction on $2 \mathrm{~h}$ plasma glucose levels in Greenlandic individuals into context, we looked up the reported effect of lifestyle intervention on $2 \mathrm{~h}$ plasma glucose levels in previously conducted landmark studies. Specifically, the results of the Finish Diabetes Prevention Study showed that 1 year of intensive lifestyle intervention, including $30 \mathrm{~min}$ of moderate physical activity per day, diet changes and reduction of body weight, reduced $2 \mathrm{~h}$ plasma glucose levels by $\sim 0.6 \mathrm{mmol} / 1$ more in the intervention group compared with the control group [11]. After 3 years of intensive lifestyle intervention, the difference in $2 \mathrm{~h}$ plasma glucose levels between the intervention group and the control group was reduced to $\sim 0.4 \mathrm{mmol} / 1$ [11]. Remarkably, we derived a similar effect size for isolated habitual physical activity in an epidemiological setting. The large effect size of the TBC1D4physical activity interaction on $2 \mathrm{~h}$ plasma glucose and the high frequency of the TBC1D4 p.Arg684Ter loss-offunction variant in the indigenous Arctic population enables a direct translation of the benefit of physical activity to tailored reduction of postprandial glucose levels for a substantial part of the population (i.e. the homozygous variant carriers).

We found indication that physical activity may have a greater beneficial effect on some (i.e. type 2 diabetes, waist/ hip ratio and insulin sensitivity index) but not all of the tested secondary outcomes in homozygous TBC1D4 p.Arg684Ter loss-of-function carriers, compared with heterozygous carriers and non-carriers. However, the results relating to secondary outcomes need to be interpreted with caution since we present results without adjustment for multiple testing. Long-term follow-up is needed before being able to make conclusions on whether there is increased risk for CVD among TBC1D4 risk variant carriers and whether physical activity may have beneficial effects on other cardiometabolic risk traits.

The discovery of gene-physical activity interactions on diabetes outcomes is challenging in commonly studied populations (such as European and Asian) and requires large sample sizes, acquired through large research biobanks or large-scale multicentre meta-analysis, that often exceed the sample size needed to identify main effects of genetic variants [21]. Therefore, our results add to the growing literature on genetic analysis in population isolates, such as the Greenlandic Inuit population. Such studies can provide a powerful approach to identify genetic variants, identify gene-environment interactions with clinical relevance, and gain knowledge into the molecular mechanisms of common disease traits [27].

Our finding of the physical activity-induced reduction of postprandial glucose levels in quasi muscle-specific human knockouts carrying the TBC1D4 p.Arg684Ter variant may pave the way towards new insights into molecular mechanisms. The variant confers loss of expression of a long TBC1D4 isoform in skeletal muscle, while other insulinsensitive tissues display normal expression of a short TBC1D4 isoform [4]. As a consequence, skeletal muscle expression levels of the long TBC1D4 isoform mRNA and protein are decreased with increasing number of TBC1D4 p.Arg684Ter risk alleles, with homozygous carriers having practically no expression [4]. Our previous study of the human homozygous TBC1D4 p.Arg684Ter variant carriers [4] and studies in Tbcld4-knockout mice unequivocally suggest that lack of TBC1D4 lowers total GLUT4 protein content in specific muscle fibre types [28-31]. In addition, insulinstimulated translocation of the GLUT4 transporter proteins to the plasma membrane might be compromised, albeit not to the same extent in different muscle fibre types [31]. The combination of lowered GLUT4 content and compromised insulin-stimulated GLUT4 translocation is suggested to lead to the postprandial hyperglycaemia and hyperinsulinaemia observed in the human homozygous TBC1D4 p.Arg684Ter variant carriers $[4,5]$. We speculate that the benefit of physical activity on lowering postprandial glucose levels in homozygous muscle-specific TBC1D4 p.Arg684Ter loss-of-function carriers might be mediated via a TBC1D4-independent muscle-specific pathway and/or tissue crosstalk from muscle to other tissues that do have an intact TBC1D4 signalling pathway. One recent example of a study pointing towards inter-organ crosstalk showed that exercise training improves glucose tolerance through crosstalk between muscle and adipose tissue through an exercise-induced adipokine [32]. It may also be possible that a change in TBC1D1, which has also been implicated in contraction induced glucose uptake [33, 34], may contribute to the enhanced response to physical activity in homozygous carriers of the TBC1D4 p.Arg684Ter loss-of-function variant.

We performed several sensitivity analyses that strengthen our findings. In the subset of individuals for whom we had information on objectively assessed physical activity we confirmed the direction and magnitude of the interaction effect. The lack of statistical significance in this sensitivity 
analysis is clearly a power issue, considering the loss, overall, of 1267 individuals (including 53 homozygous TBC1D4 p.Arg684Ter variant carriers) compared with the analysis based on questionnaire-derived physical activity. Interestingly, the commonly used additive model showed that the TBC1D4 p.Arg684Ter-physical activity interaction also had a significant effect on $2 \mathrm{~h}$ plasma glucose levels in the subset of individuals with objectively assessed physical activity. However, the magnitude of the interaction effect was overall smaller than the interaction effect derived from the primary model, likely due to heterozygous TBC1D4 p.Arg684Ter variant carriers that are accounted for in an additive interaction model but not in the primary recessive model. Compared with homozygous carriers, in heterozygous carriers (more common in the Greenlandic population [4]) the TBC1D4 p.Arg684Ter variant has less of an effect on metabolic traits, explaining the increased statistical power by applying an additive interaction model. Finally, the presented results are not confounded by population structure, the degree of admixture or relatedness among the Greenlandic population.

We were limited to the analysis of epidemiological data collected as part of a general health survey of the Greenlandic population and therefore the reported effect sizes need careful interpretation. To quantify the clinical impact of physical activity in carriers of the TBC1D4 p.Arg684Ter lossof-function variant and, based on this, recommend personalised treatment, an exercise-training intervention trial specifically targeted to Arctic individuals should be performed. Such a trial might include detailed OGTT measures of glucose homeostasis and type 2 diabetes remission as primary outcomes, as well as collection of tissue biopsies for functional and biochemical analysis. If designed properly, studies of human TBC1D4 p.Arg684Ter loss-of-function variant carriers may also provide information on molecular mechanisms of the role of TBC1D4 in the beneficial effects of physical activity and determine whether TBC1D1 may play a contributary role in the enhanced response to physical activity in homozygous carriers of the TBC1D4 p.Arg684Ter lossof-function variant. We measured circulating glucose levels during an OGTT and interpreted our results as if skeletal muscle is the major tissue involved in exercise-induced priming of glucose uptake. However, we cannot exclude that other organs such as adipose tissue may play a role in the improved glucose tolerance in homozygous TBC1D4 variant carriers who spend more time performing physical activity.

In summary, the results of our study suggest that regular moderate physical activity produces a clinically relevant improvement in postprandial glucose levels in indigenous Arctic individuals carrying two copies of the TBC1D4 p.Arg684Ter loss-of-function variant. This provides a rationale to implement lifestyle intervention therapy by motivating a physically active lifestyle among homozygous carriers of this variant, constituting $3.8 \%$ of the population.
Supplementary Information The online version contains peer-reviewed but unedited supplementary material available at https://doi.org/10.1007/ s00125-021-05461-z.

Acknowledgements We thank all participants in the study. We thank B. Carstensen (Steno Diabetes Center Copenhagen, Gentofte, Denmark) for his expertise and assistance throughout the statistical aspects of the geneenvironment interaction model and also thank I. Olesen (National Institute of Public Health, University of Southern Denmark, Odense, Denmark and University of Nuuk, Greenland). Parts of this work were presented as an oral presentation at the 52nd EASD Conference in Munich, Germany, 12-16 September 2016 and as an oral presentation at the NunaMed meeting in Nuuk, Greenland, 1-3 October 2016.

Data availability Relevant data for the present study are within the presented article and in the ESM. The Greenlandic Cardio-Metabochip data for the Inuit Health in Transition study has been deposited at the European Genomephenome Archive (https://www.ebi.ac.uk/ega/dacs/EGAC00001000736) under accession EGAD00010001428. If you wish to see additional data, the authors confirm that, for approved reasons, some access restrictions apply. Data are available from the Novo Nordisk Foundation Center for Basic Metabolic Research; authors may be contacted at torben.hansen@sund.ku.dk.

Funding The collection of data for the Greenland Inuit Cohort was supported by Karen Elise Jensen's Foundation, NunaFonden, the Medical Research Council of Denmark, the Medical Research Council of Greenland and the Commission for Scientific Research in Greenland. The Novo Nordisk Foundation Center for Basic Metabolic Research is an independent research centre at the University of Copenhagen and is partly funded by an unrestricted donation from the Novo Nordisk Foundation. This project was also supported by funds from the Danish Council for Independent Research (Medical Sciences), the Steno Diabetes Center, the Villum Foundation, the Danish Diabetes Academy, the Novo Nordisk Foundation (NNF14OC0013057, NNF15OC0017918 and NNF16OC0019986) and the Lundbeck Foundation. This work was supported by the Medical Research Council UK (MC UU 12015/3 to SB). HAH received grants from the European Foundation for the Study of Diabetes and Novo Nordisk. None of the funding agencies had any role in the study design or in the collection or interpretation of the data.

Authors' relationships and activities $\mathrm{HAH}$ has served as consultant at Bayer AG, MEJ has received research grants from Amgen, SanofiAventis, AstraZeneca and Boehringer Ingelheim. MEJ holds shares in Novo Nordisk. The remaining authors declare no relationships or activities that might bias, or be perceived to bias, their work.

Contribution statement TMS, EJ, AA and TH helped to conceive and design the analysis. TMS and EJ performed the analysis. TMS, EJ and $\mathrm{AC}$ drafted the manuscript. $\mathrm{AA}, \mathrm{HAH}$ and $\mathrm{TH}$ supported drafting of the manuscript. IP, MEJ and PB collected human data and contributed to discussion. SB contributed with expertise regarding the physical activity measures. IM helped to design the technical phrases of the manuscript. AC, CS, HAH, NG, JMK, JW and OP contributed to discussion. TMS and TH had full access to all data and were responsible for the decision to submit for publication. All authors interpreted the results, edited and revised the manuscript and finally read and approved the final version of the manuscript. TMS, EJ and TH had full access to all data, are the guarantors of this work and were responsible for the decision to submit for publication.

Open Access This article is licensed under a Creative Commons Attribution 4.0 International License, which permits use, sharing, adaptation, distribution and reproduction in any medium or format, as long as you give appropriate credit to the original author(s) and the source, 
provide a link to the Creative Commons licence, and indicate if changes were made. The images or other third party material in this article are included in the article's Creative Commons licence, unless indicated otherwise in a credit line to the material. If material is not included in the article's Creative Commons licence and your intended use is not permitted by statutory regulation or exceeds the permitted use, you will need to obtain permission directly from the copyright holder. To view a copy of this licence, visit http://creativecommons.org/licenses/by/4.0/.

\section{References}

1. Jørgensen ME, Borch-Johnsen K, Stolk R, Bjerregaard P (2013) Fat distribution and glucose intolerance among Greenland Inuit. Diabetes Care 36(10):2988-2994. https://doi.org/10.2337/dc122703

2. Bjerregaard P (2011) Inuit health in transition: Greenland survey 2005-2010: population sample and survey methods. National Institute of Public Health Copenhagen

3. Chen L, Magliano DJ, Zimmet PZ (2011) The worldwide epidemiology of type 2 diabetes mellitus - present and future perspectives. Nat Rev Endocrinol 8(4):228-236

4. Moltke I, Grarup N, Jørgensen ME et al (2014) A common Greenlandic TBC1D4 variant confers muscle insulin resistance and type 2 diabetes. Nature 512(7513):190-193. https://doi.org/ 10.1038 /nature13425

5. Manousaki D, Kent JW, Haack K et al (2016) Toward precision medicine: TBC1D4 disruption is common among the Inuit and leads to underdiagnosis of type 2 diabetes. Diabetes Care 39(11):1889-1895

6. Fitipaldi H, McCarthy MI, Florez JC, Franks PW (2018) A global overview of precision medicine in type 2 diabetes. Diabetes 67 : 1911-1922. https://doi.org/10.2337/dbi17-0045

7. Mohan V, Radha V (2019) Precision diabetes is slowly becoming a reality. Med Princ Pract 28(1):1-9. https://doi.org/10.1159/ 000497241

8. Sano H, Kane S, Sano E et al (2003) Insulin-stimulated phosphorylation of a Rab GTPase-activating protein regulates GLUT4 translocation. J Biol Chem 278(17):14599-14602. https://doi.org/10. 1074/jbc.C300063200

9. Wang HY, Ducommun S, Quan C et al (2013) AS160 deficiency causes whole-body insulin resistance via composite effects in multiple tissues. Biochem J 449(2):479-489. https://doi.org/10.1042/ BJ20120702

10. Vind BF, Pehmøller C, Treebak JT et al (2011) Impaired insulininduced site-specific phosphorylation of TBC1 domain family, member 4 (TBC1D4) in skeletal muscle of type 2 diabetes patients is restored by endurance exercise-training. Diabetologia 54(1):157167. https://doi.org/10.1007/s00125-010-1924-4

11. Lindström J, Louheranta A, Mannelin M et al (2003) The Finnish diabetes prevention study (DPS): lifestyle intervention and 3-year results on diet and physical activity. Diabetes Care 26(12):32303236. https://doi.org/10.2337/diacare.26.12.3230

12. Ostman C, Smart NA, Morcos D, Duller A, Ridley W, Jewiss D (2017) The effect of exercise training on clinical outcomes in patients with the metabolic syndrome: a systematic review and meta-analysis. Cardiovasc Diabetol 16(1):110

13. Alberti KG, Zimmet PZ (1997) Definition, diagnosis and classification of diabetes mellitus and its complications part 1: diagnosis and classification of diabetes mellitus provisional report of a WHO consultation. Diabet Med 15(7):539-553

14. Dahl-Petersen IK, Hansen AW, Bjerregaard P, Jørgensen ME, Brage S (2013) Validity of the international physical activity questionnaire in the arctic. Med Sci Sports Exerc 45(4):728-736
15. Dahl-Petersen IK, Bjerregaard P, Brage S, Jørgensen ME (2013) Physical activity energy expenditure is associated with 2-h insulin independently of obesity among Inuit in Greenland. Diabetes Res Clin Pract 102(3):242-249. https://doi.org/10.1016/j.diabres.2013.10.004

16. Brage S, Brage N, Franks PW et al (2004) Branched equation modeling of simultaneous accelerometry and heart rate monitoring improves estimate of directly measured physical activity energy expenditure. J Appl Physiol 96(1):343-351. https://doi.org/10. 1152/japplphysiol.00703.2003

17. Ainsworth BE, Haskell WL, Herrmann SD et al (2011) 2011 compendium of physical activities: a second update of codes and MET values. Med Sci Sports Exerc 43(8):1575-1581. https://doi. org/10.1249/MSS.0b013e31821 ece12

18. Voight BF, Kang HM, Ding J et al (2012) The metabochip, a custom genotyping array for genetic studies of metabolic, cardiovascular, and anthropometric traits. PLoS Genet 8(8):e1002793. https://doi.org/10.1371/journal.pgen.1002793

19. Chang CC, Chow CC, Tellier LC, Vattikuti S, Purcell SM, Lee JJ (2015) Second-generation PLINK: rising to the challenge of larger and richer datasets. Gigascience 4:7

20. Chen H, Wang C, Conomos MP et al (2016) Control for population structure and relatedness for binary traits in genetic association studies via logistic mixed models. Am J Hum Genet 98(4):653666. https://doi.org/10.1016/j.ajhg.2016.02.012

21. Kilpeläinen TO, Franks PW (2014) Gene-physical activity interactions and their impact on diabetes. Med Sport Sci 60:94-103

22. Wong MY, Day NE, Luan JA, Chan KP, Wareham NJ (2003) The detection of gene-environment interaction for continuous traits: should we deal with measurement error by bigger studies or better measurement? Int J Epidemiol 32(1):51-57. https://doi.org/10. 1093/ije/dyg002

23. Kilpeläinen TO, Qi L, Brage S et al (2011) Physical activity attenuates the influence of FTO variants on obesity risk: a meta-analysis of 218,166 adults and 19,268 children. PLoS Med 8(11):1543

24. Langenberg C, Sharp SJ, Franks PW et al (2014) Gene-lifestyle interaction and type 2 diabetes: the EPIC interact case-cohort study. PLoS Med 11(5):e1001647

25. Scott RA, Chu AY, Grarup N et al (2012) No interactions between previously associated 2-hour glucose gene variants and physical activity or BMI on 2-hour glucose levels. Diabetes 61(5):1291-1296

26. Klimentidis YC, Chen Z, Arora A, Hsu C-H (2014) Association of physical activity with lower type 2 diabetes incidence is weaker among individuals at high genetic risk. Diabetologia 57(12): 2530-2534. https://doi.org/10.1007/s00125-014-3380-z

27. Grarup N, Moltke I, Albrechtsen A, Hansen T (2015) Diabetes in population isolates: lessons from Greenland. Rev Diabet Stud 12(3-4):320-329

28. Chadt A, Immisch A, de Wendt C et al (2015) Deletion of both RabGTPase-activating proteins TBC1D1 and TBC1D4 in mice eliminates insulin- and AICAR-stimulated glucose transport [corrected]. Diabetes 64(3):746-759. https://doi.org/10.2337/db14-0368

29. Lansey MN, Walker NN, Hargett SR, Stevens JR, Keller SR (2012) Deletion of Rab GAP AS160 modifies glucose uptake and GLUT4 translocation in primary skeletal muscles and adipocytes and impairs glucose homeostasis. Am J Physiol Endocrinol Metab 303(10):E1273E1286. https://doi.org/10.1152/ajpendo.00316.2012

30. Hong YW, Serge D, Chao Q et al (2013) AS160 deficiency causes whole-body insulin resistance via composite effects in multiple tissues. Biochem J 449(2):479-489

31. Hargett SR, Walker NN, Keller SR (2016) Rab GAPs AS160 and Tbc1d1 play nonredundant roles in the regulation of glucose and energy homeostasis in mice. Am J Physiol Endocrinol Metab 310(4):E276-E288. https://doi.org/10.1152/ajpendo.00342.2015

32. Takahashi H, Alves CRR, Stanford KI et al (2019) TGF- $\beta 2$ is an exercise-induced adipokine that regulates glucose and fatty acid 
metabolism. Nat Metab 1(2):291-303. https://doi.org/10.1038/ s42255-018-0030-7

33. Stöckli J, Meoli CC, Hoffman NJ et al (2015) The RabGAP TBC1D1 plays a central role in exercise-regulated glucose metabolism in skeletal muscle. Diabetes 64(6):1914-1922. https://doi. org/10.2337/db13-1489

34. Whitfield J, Paglialunga S, Smith BK et al (2017) Ablating the protein TBC1D1 impairs contraction-induced sarcolemmal glucose transporter 4 redistribution but not insulin-mediated responses in rats. J Biol Chem 292(40):16653-16664. https://doi.org/10.1074/ jbc.M117.806786

Publisher's note Springer Nature remains neutral with regard to jurisdictional claims in published maps and institutional affiliations. 